\title{
Nouvelle approche par l'archéozoologie dans les nécropoles antiques d'Egiin Gol et de Gol Mod (Mongolie)
}

Hélène Martin, Guilhem André et Jean-Paul Desroches

\section{(2) OpenEdition \\ Journals}

Édition électronique

URL : https://journals.openedition.org/archeopages/808

DOI : 10.4000/archeopages. 808

ISSN : 2269-9872

Éditeur

INRAP - Institut national de recherches archéologiques préventives

Édition imprimée

Date de publication : 1 octobre 2010

Pagination : 114-123

ISSN : 1622-8545

\section{Référence électronique}

Hélène Martin, Guilhem André et Jean-Paul Desroches, « Nouvelle approche par l'archéozoologie dans les nécropoles antiques d'Egiin Gol et de Gol Mod (Mongolie) », Archéopages [En ligne], Hors-série 2 I 2010, mis en ligne le 01 octobre 2010, consulté le 23 février 2023. URL : http://

journals.openedition.org/archeopages/808; DOI : https://doi.org/10.4000/archeopages.808 


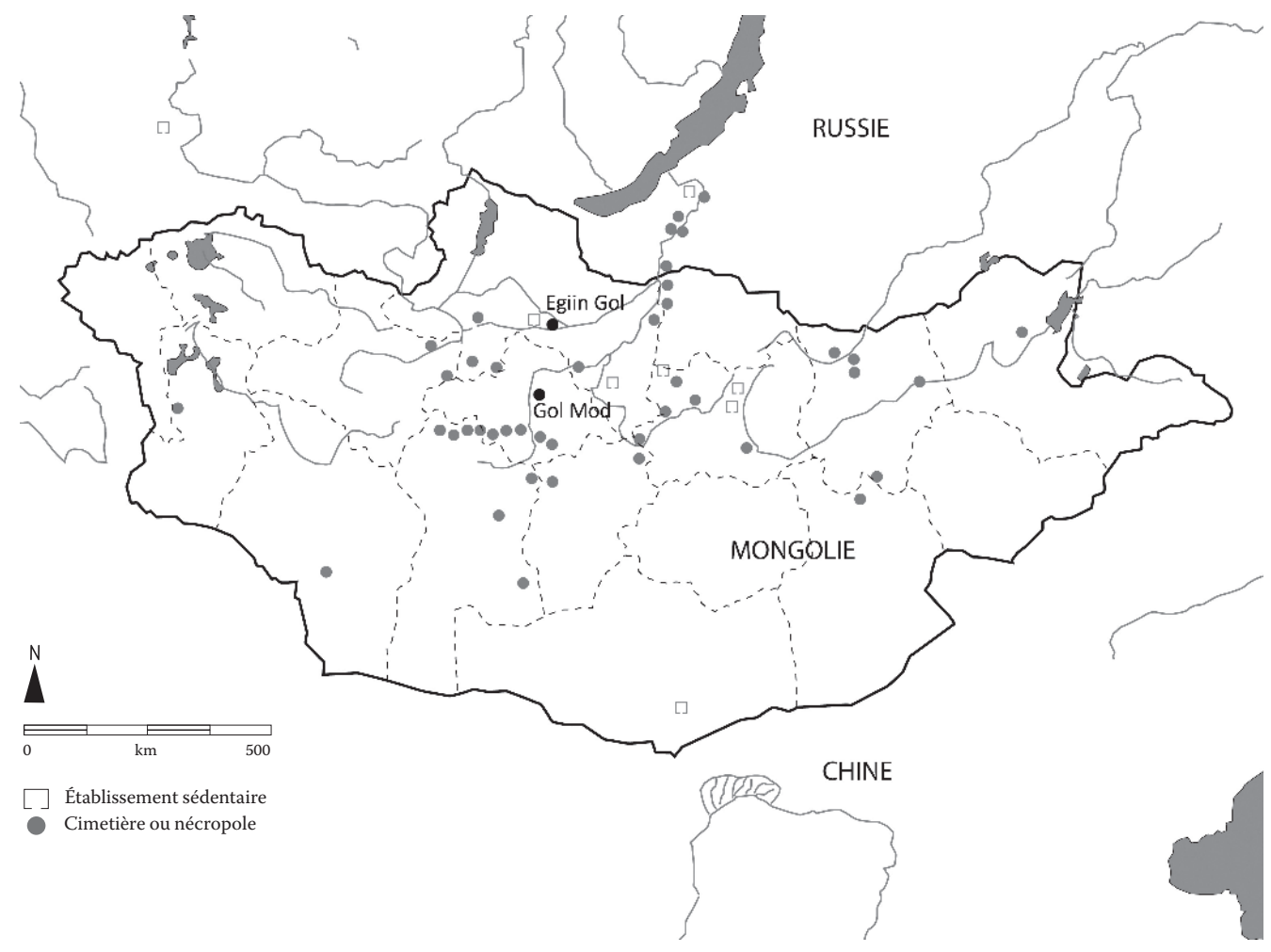

$\exists$

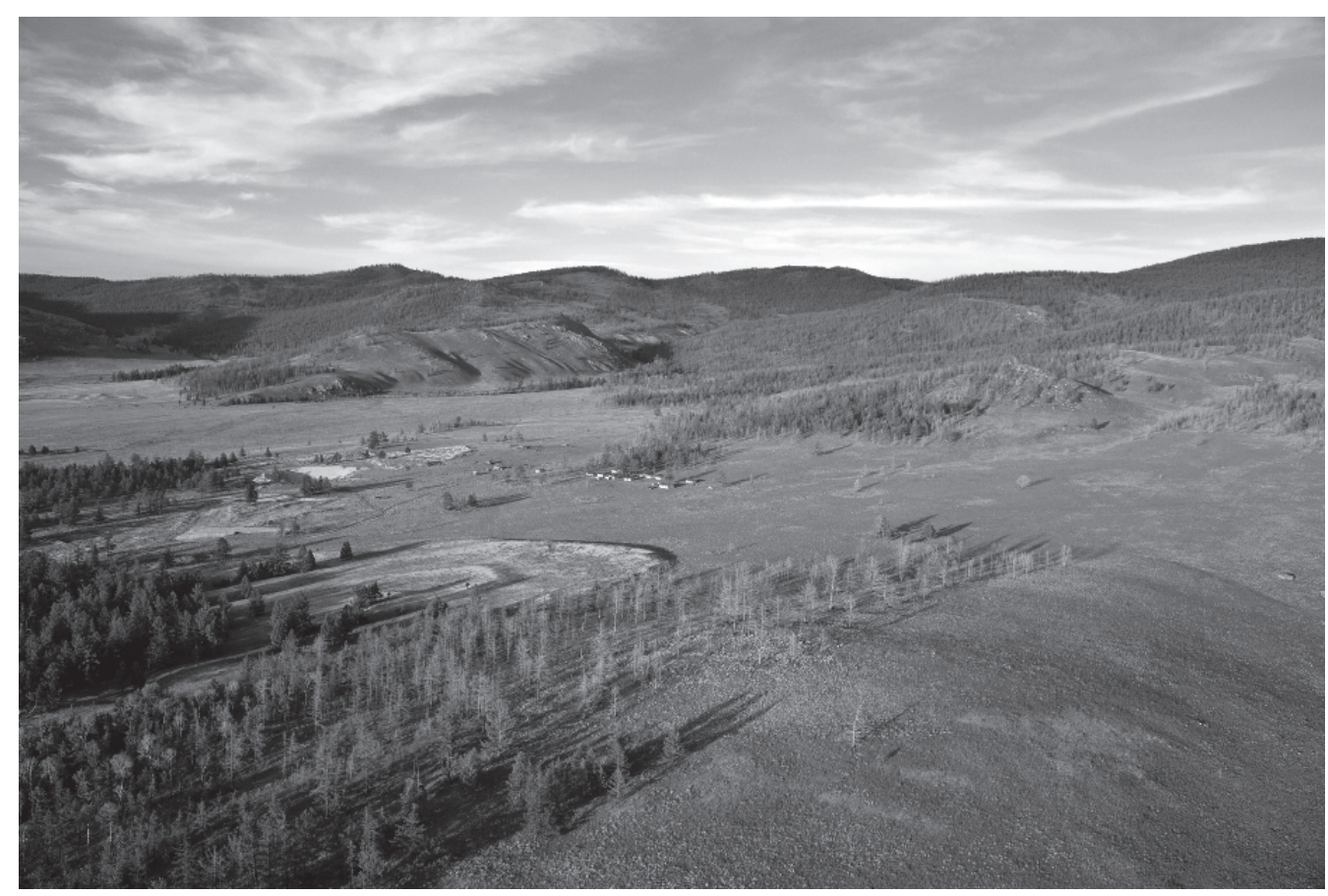

[Fig.1] Carte des sites archéologiques xiongnu de Mongolie.

[Fig.2] Vue aérienne du site de Gol Mod, Arkhangaï, Mongolie. 
institutions impliquées dans l'archéologie, comme l'Université hébraïque de Jérusalem, l'Université

Ben Gourion et l'Université de Tel-Aviv. Le centre français de Jérusalem (CRFJ) a accueilli de nombreux chercheurs français en archéologie et en anthropologie. Ils ont été intégrés à de nombreux projets sur le long terme, concernant diverses thématiques. Pierre de Miroschedgi a dirigé, avec l'Université hébraïque, la fouille de Tel Yarmouth, l'un des sites majeurs du Bronze ancien en Israël. François Valla a mené plusieurs projets avec différentes institutions (comme l'Université hébraïque de Jérusalem) sur la terrasse d'Hayonim et, avec l'Université Ben Gourion, une enquête préhistorique dans le nord du Néguev; plus récemment encore, il a participé aux fouilles de l'Ain Mallaha, en collaboration étroite avec l'Israel Antiquities Authority (IAA). Monique Lechevallier a fouillé le site de Hatula avec l'Université de Haïfa. Bernard Vandermeersch, Anne-Marie Tallier et d'autres chercheurs français ont travaillé sur les occupations de l'homme de Néandertal dans les grottes du Carmel et en Galilée, en collaboration avec les institutions israéliennes.

Le récent accord de coopération scientifique internationale entre l'IA A et l'Inrap a été une plateforme importante pour envisager une collaboration sur des projets archéologiques en Israël. Dans le cadre de cet accord, les chercheurs des deux instituts se doivent de «partager leurs compétences dans le domaine de l'archéologie préventive, de participer à des opérations archéologiques et de faciliter les collaborations scientifiques mutuelles dans les recherches archéologiques qu'ils effectuent ». Sur la base de cet accord, deux projets archéologiques ont été menés récemment en Israël: la reprise de l'étude du site de Beisamoun (cf. article p. 135) et le projet Césarée (cf. p. 103).

La fouille de Beisamoun est un projet codirigé par l'équipe d'experts de trois instituts : le CNRS, représenté par Fanny Bocquentin, l'IAA, par Hamoudi Khalaily, et l'Inrap, par Nicolas Samuelian. Le site de Beisamoun (9000-7900 avant notre ère) est situé dans la partie occidentale de la vallée de Hula, et ses vestiges s'étendent sur une superficie d'environ 12 ha. Le site a été sondé en 1965 par des archéologues français et les structures découvertes (murs, planchers en plâtre et éléments lithiques) ont permis de le dater du Néolithique précéramique B. Il n'y a plus eu d'investigations archéologiques de 1972 à 2007. Le projet actuel vise à étudier les techniques architecturales de la fin du Néolithique précéramique, à analyser systématiquement les assemblages de silex et de faune ; il offre aussi une occasion rare d'étudier, dans une perspective diachronique, la dynamique de l'occupation des sites d'exploitation de l'environnement et la transmission culturelle au sein du bassin de Hula. Les diverses compétences, expériences et méthodes de fouille des membres de l'équipe sont un atout majeur pour atteindre ces objectifs. Le projet n'en est qu'à ses débuts et les possibilités de découvertes sont nombreuses, comme le montrent les rapports des premières saisons. Nicolas Samuelian supervise l'une des deux zones de fouilles tout en étant responsable de l'enregistrement des données. C'est en s'appuyant sur son expérience qu'ont pu être menés un grand décapage suivant les méthodes développées par les archéologues de l'Inrap et une analyse stratigraphique très fine, deux réalisations qui contribuent à la compréhension des vestiges néolithiques.

La fouille à Césarée est un autre exemple d'une collaboration tripartite entre l'IAA, représenté par Hervé Barbé, l'Inrap, par Jocelyn Martineau et Fabien Sanz-Pascual, et l'Université de Nantes, sous la direction de Nicolas Faucherre. Il s'agit d'étudier l'architecture mamelouke du château, de la ville, du mur d'enceinte et du port. La responsabilité des archéologues de l'Inrap porte autant sur le relevé des vestiges archéologiques que sur leur étude et l'élaboration d'hypothèses de reconstitution.

Pour l'IAA, les recherches menées sur ces sites fondamentaux sont primordiales pour la compréhension des périodes préhistoriques et historiques en Israël. Cette collaboration est également précieuse car elle offre la possibilité aux diverses institutions partenaires de combiner leurs ressources et leurs connaissances pour la promotion de la science archéologique.

\section{Nouvelle approche par l'archéozoologie dans les nécropoles antiques d'Egiin Gol et de Gol Mod (Mongolie)}

\author{
Hélène Martin \\ Inrap, Archéozoologue et UMR 5608 " Traces» \\ Guilhem André \\ Mission archéologique française en Mongolie (MAFM)/ \\ Musée des Arts asiatiques-Guimet \\ Jean-Paul Desroches \\ MAFM/Musée des Arts asiatiques-Guimet
}

P résupposés sur la civilisation xiongnu. La connaissance de la civilisation xiongnu ( $\mathrm{III}^{\mathrm{e}}$ siècle avant notre ère - $\mathrm{III}^{\mathrm{e}}$ de notre ère) de Mongolie s'est longtem ps fondée sur les informations textuelles émanant de leurs voisins chinois, chroniqueurs de la dynastie des Han (206 avant notre ère-220 de notre ère) et des suivantes. Les annales historiques de l'Empire constituent en effet des sources primordiales, mais l'objectivité de certains auteurs antiques demeure aléatoire, de même que la fiabilité de leurs informations, eut égard aux relations conflictuelles entre ces deux puissances voisines : les lettrés se trouvaient confrontés à des problèmes d'accès aux données, de transcription et de transmission de leurs textes.

Pour une majorité de spécialistes, l'organisation des Xiongnu adoptait la forme d'une confédération de tribus occupant un territoire qui s'étendait, à l'apogée de leur puissance, de l'Altaï à la Mandchourie et du Baïkal à la boucle du fleuve Jaune [Fig.1]. À la tête de cette confédération, leurs 


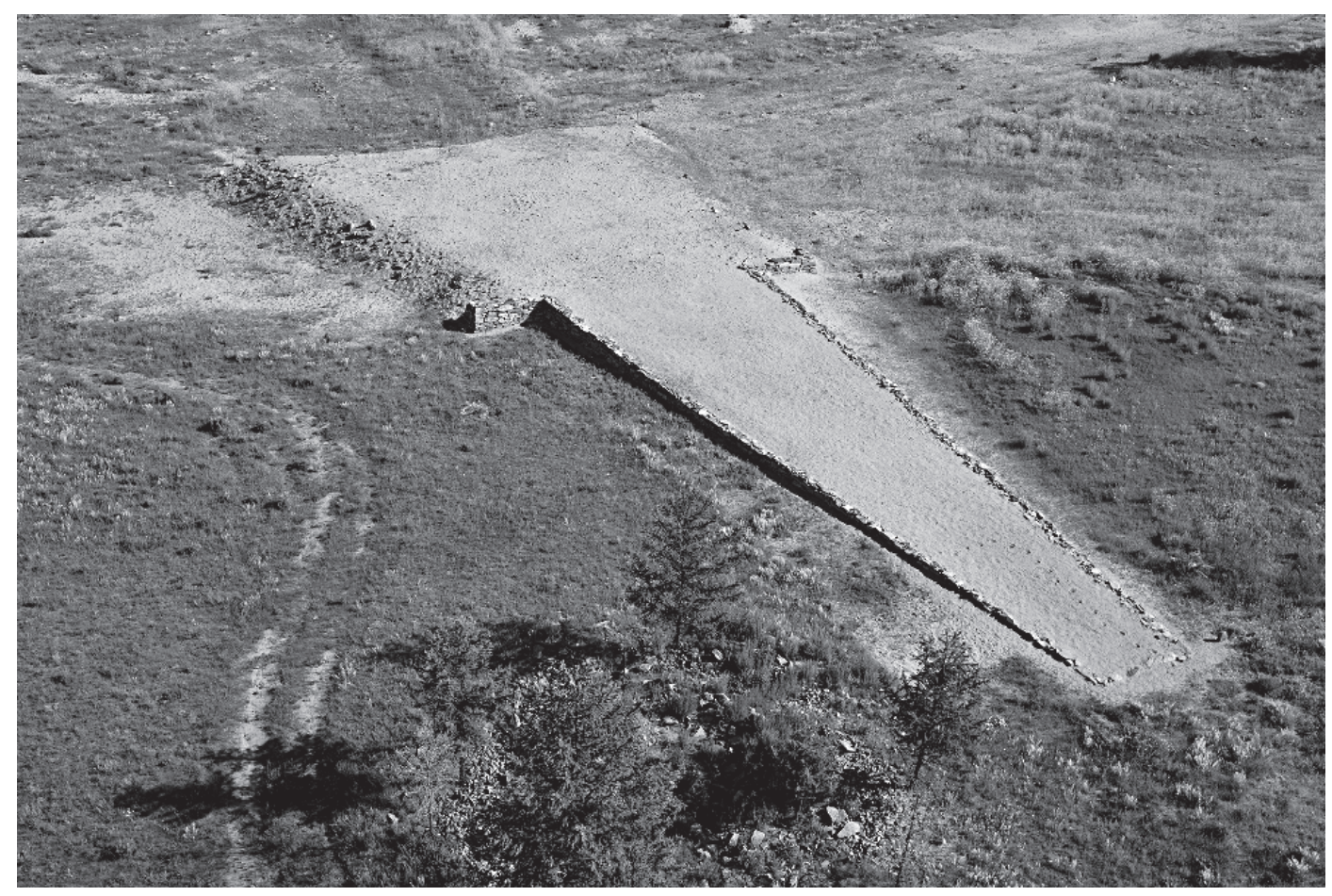

曲

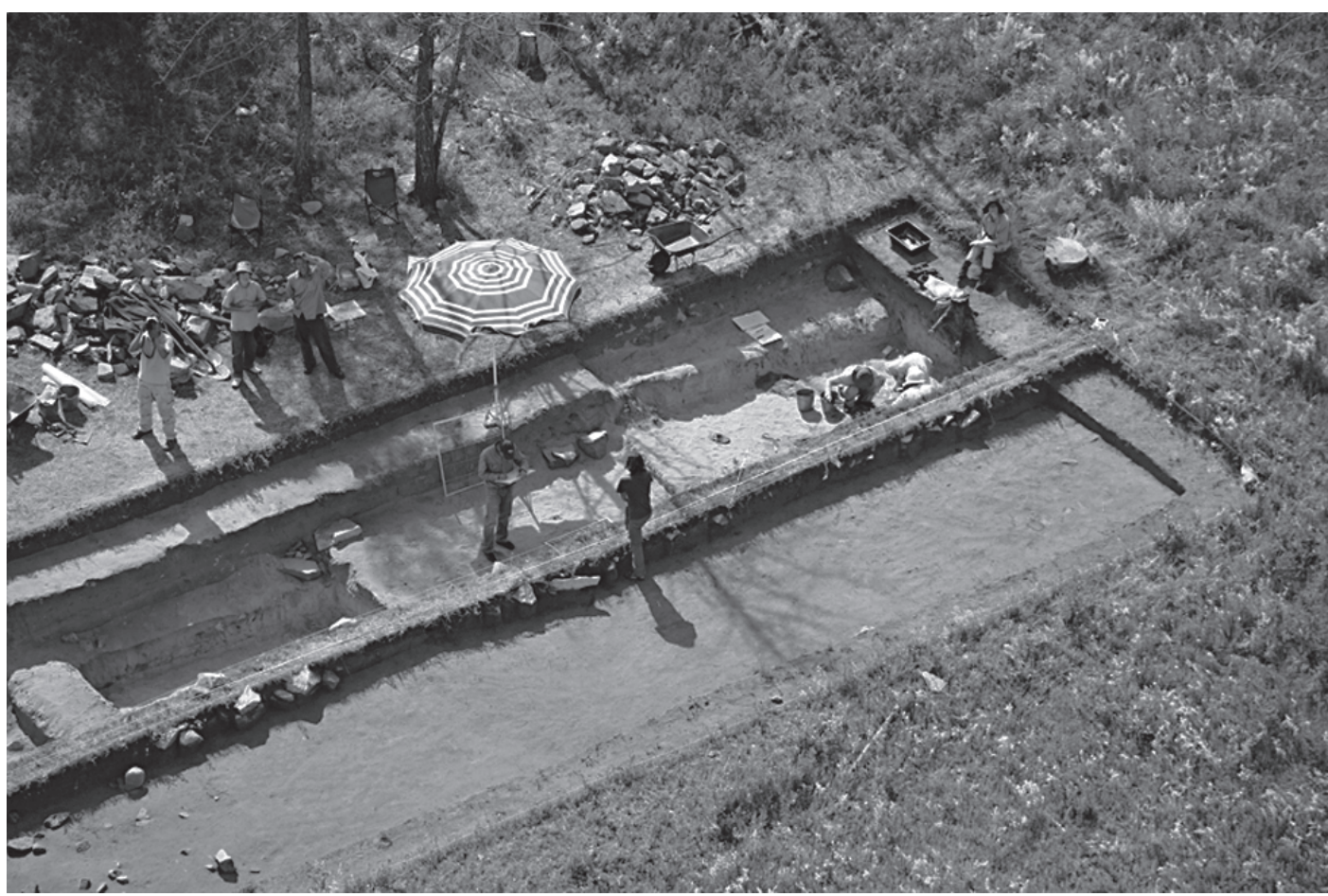

[Fig.3] Vue aérienne de la tombe T1 reconstruite à l'identique, Gol Mod, Arkhangaï, Mongolie, $\mathrm{I}^{\mathrm{er}}$ siècle de notre ère.
[Fig.4] Fouilles de sépultures adventices à la tombe T416, Gol Mod, été 2008. 
souverains et chefs de clans, les chanyu, détenaient un pouvoir de nature à la fois administratif, militaire et - peut-être - religieux. L'hypothèse répandue consiste à considérer ce peuple comme une organisation d'éleveurs nomades, assez comparable à celle des populations évoluant actuellement dans la steppe. Leur économie n'aurait toutefois pas seulement reposé sur l'élevage, mais aurait été soutenue par des pillages réguliers, pratiqués lors d'incursions chez leurs voisins sédentaires han, et elle aurait été complétée par une agriculture vivrière à petite échelle. Ce schéma se voit désormais modifié, voire remis en question, par les travaux archéologiques récents en Mongolie, en particulier à partir des informations livrées, sur les pratiques funéraires, par l’archéozoologie.

La Mission archéologique française en Mongolie. Soutenue par la Commission des fouilles du ministère des Affaires étrangères et européennes, la MAFM commence en 1993 à œuvrer en collaboration avec l'Académie des Sciences de Mongolie. Bien qu'assez nombreuses, les recherches précédentes, généralement sporadiques, se sont concentrées sur un petit nombre des quelque 70 sites xiongnu du pays. La MAFM souhaitait documenter la culture xiongnu à travers un programme principalement, mais pas uniquement, dédié au domaine funéraire autour des problématiques liées aux modes de vie et aux circuits économiques de ces sociétés. Dans notre approche pluridisciplinaire, la lecture critique et comparée des textes historiques chinois avec les découvertes archéologiques constituait un point essentiel car très complémentaire. Explorant principalement deux sites, la mission s'est installée dans la province de Bulgan, dans le nord du pays, pour une fouille de sauvetage le long de la vallée d'Egiin Gol dans le district de Khutag-Öndör. Jusqu'en 1999, son activité a été exclusivement consacrée à la fouille de 89 des 116 inhumations d'un cimetière de nature plébéienne dont une trentaine ont pu être datées par analyse au radiocarbone. Parmi les autres résultats obtenus sur le mobilier, citons en particulier les premiers examens par analyse ADN des restes humains xiongnu, effectués en vue d'identifier d'éventuels liens familiaux entre les individus. C'était la première fois, en Mongolie, qu'une fouille était pratiquée à l'échelle de l'ensemble d'un site xiongnu.

En 2000, la mission s'est installée sur un second site, à Gol Mod, dans la province d'Arkhangaï, l'une des six nécropoles aristocratiques xiongnu localisées en Mongolie. Gol Mod se situe sur la rive méridionale de la rivière Khünüi, au pied de montagnes appartenant à la chaîne de Khangaï [Fig.2], aux pentes couvertes de mélèzes, en contraste fort avec la végétation plus aride des vallées. L'emplacement avait été identifié en 1955 par l'archéologue mongol Ts. Dorjsüren, qui organisa deux campagnes successives les années suivantes, lors desquelles il étudia 26 tombes et débutala fouille d'un monument de $70 \mathrm{~m}$ de long, la T1, un travail inachevé qui sera repris par la MAFM. Cette nécropole regroupe une concentration de complexes funéraires caractérisés par un axe sud-nord et composés d'inhumations principales flanquées d'une série de sépultures adventices [Fig. 3, 4 et 5].

En surface, les premières comportent une terrasse en pierre sèche quadrangulaire, à laquelle conduit une allée constituant une rampe d'accès en profondeur. Les secondes, plus modestes, sont délimitées par un simple cercle de pierres. Ces deux catégories peuvent aussi être isolées et ne pas, a priori, constituer de complexes. Entre les pôles de regroupement se trouvent des zones apparemment dépourvues de vestiges anthropiques. Prospections, relevés topographiques et géophysiques sont notamment employés sur ce site de presque 4.00 ha où 393 tombes ont été identifiées, mais où la fouille s'est concentrée sur 22 inhumations, dont trois sépultures monumentales.

La steppe étant, à Gol Mod, comme dans l'ensemble de la Mongolie, un écosystème résultant de fragiles équilibres environnementaux, l'étude $\mathrm{du}$ facteur climatique et des ressources naturelles reste un élément crucial pour mieux comprendre la vie de ces populations. L'irrégularité structurelle du climat mongol et la somme de précarités qu'elle génère constituent le cadre principal du système pastoral nomade de l'essentiel du territoire mongol actuel et, suppose-t-on, antique. Une connaissance plus approfondie de l'environnement et, en particulier, du cheval, dont les restes sont souvent découverts associés au domaine funéraire, pouvait dès lors apporter une compréhension beaucoup plus précise de la société xiongnu, afin notamment de valider ou d'invalider son caractère nomade.

L'apport de l'archéozoologie. Lors de la mise au jour de restes d'animaux en contexte funéraire, il convient de définir d'une part s'ils sont associés ou non aux dépôts humains et d'autre part de préciser leur nature : offrandes offertes aux défunts, restes de repas funéraires ou représentations purement symboliques dont la fonction est d'interférer entre le monde des vivants et le monde des morts. Si la première question peut généralement se résoudre sur le terrain, voire lors de l'étude en laboratoire, la seconde est plus complexe car elle suppose l'identification de l'intention qui prélude au dépôt.

L'observation directe in situ des vestiges animaux apporte des informations essentielles quant aux différentes interactions entre l'homme et l'animal. Les données archéologiques redessinant l'architecture des tombes fouillées vont permettre à l'archéozoologue de définir la situation des restes d'animaux dans ces monuments et de préciser s'ils correspondent, ou non, à des dépôts volontaires se trouvant en position primaire ou secondaire. Pour ce faire, au-delà des connaissances relatives à l'anatomie comparée et propres à la spécialité, il est indispensable d'avoir une bonne connaissance de la décomposition des corps et de l'enregistrement de ces phénomènes dans les sédiments. Ces altérations se compliquent souvent de perturbations survenues postérieurement au dépôt et qui sont 

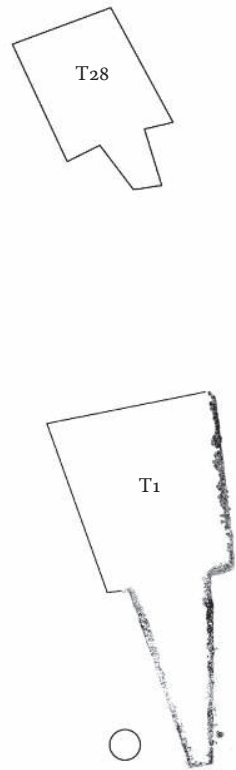

$\stackrel{\infty}{\exists}$
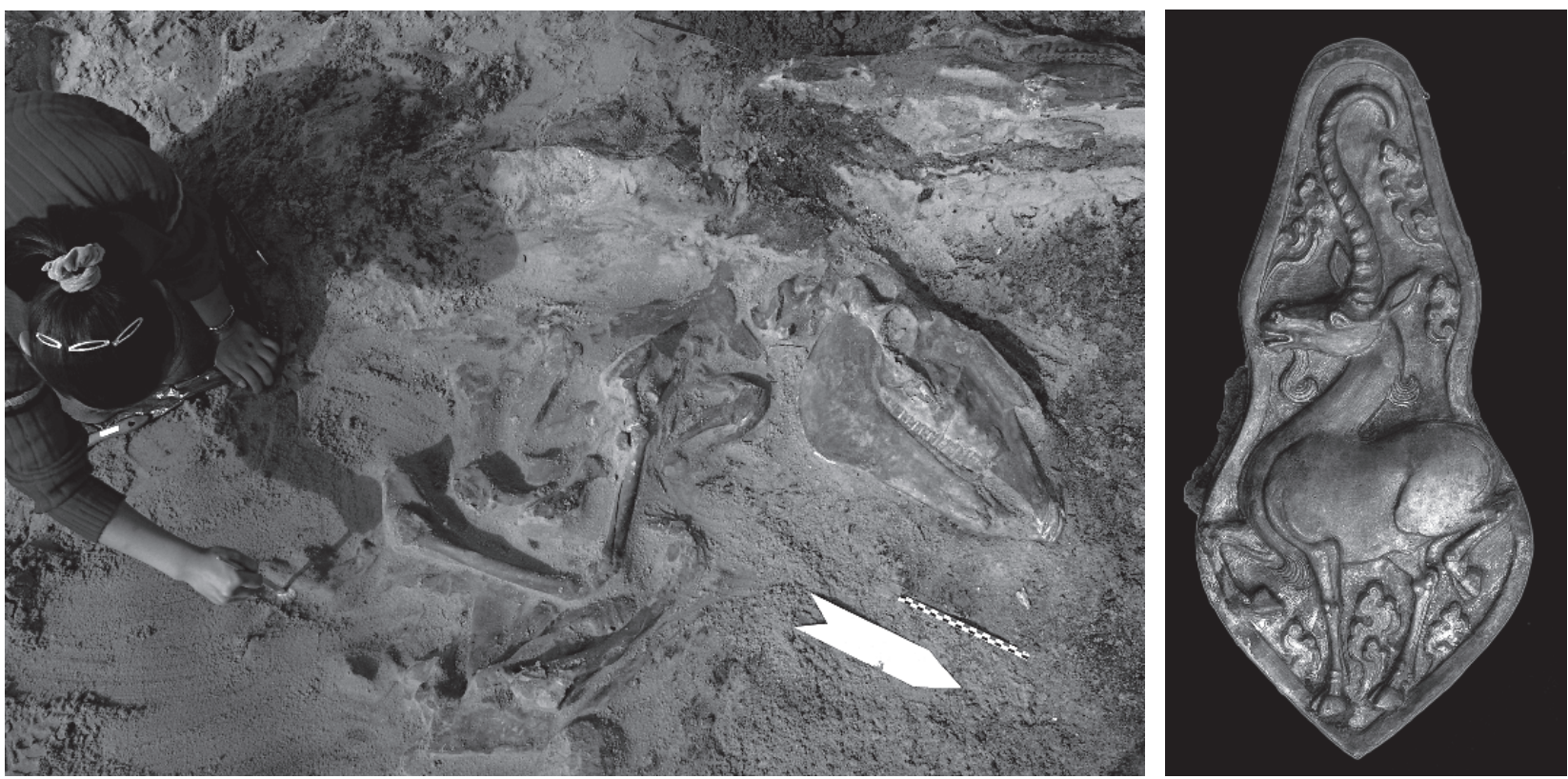

[Fig.5] Relevé de superstructures dans le secteur nord, dont la tombe $\mathrm{T} 2 \mathrm{O}$.
[Fig.6] Fouille du niveau de dépôt de restes animaux, tombe $\mathrm{T} 2 \mathrm{O}$, Gol Mod, $\mathrm{I}^{\text {er }}$ siècle de notre ère.
[Fig.7] ] Plaque décorative de harnachement à décor d'animal

fantastique, argent doré, H. 14,7 cm, tombe T20, Gol Mod, ${ }^{\text {er }}$ siècle de notre ère. 
soit l'expression de rites funéraires spécifiques à une culture, soit la conséquence de pillages dont la vocation profonde ne se limite pas nécessairement au simple larcin. La situation initiale des vestiges par rapport au corps du défunt devra donc également être déterminée avec précision. Seule une parfaite connaissance de l'évolution naturelle du squelette à travers l'identification de la taille, de l'âge et parfois du sexe de l'animal déterminé, permet de préciser si certains os, découverts isolés, ont été déposés seuls ou bien s'ils s'associaient à d'autres portions osseuses avant d'en être séparés lors de perturbations ultérieures à leur placement dans la tombe.

Il nous est apparu primordial de développer une approche relative à la saison d'abattage des animaux. Celle-ci peut-être déterminée par l'examen des anneaux de cément se formant de manière cyclique sur les racines dentaires des mammifères et suppose de disposer de spécimens suffisamment bien conservés (Le Gall et al., sous presse). Les informations résultant de ces analyses peuvent se révéler d'autant plus précieuses que, parfois, en Asie centrale, on observe un décalage entre décès et inhumation, lié à des conditions climatiques rigoureuses ou à d'autres facteurs, rituels par exemple. Ainsi, on conçoit aisément que la construction de certaines tombes, comme la sépulture T2o de Gol Mod, qui servira d'illustration à ce propos, implique un temps relativement long. Même en s'appuyant sur le concours de très nombreux participants, il n'est pas certain que l'ensemble des travaux se soient déroulés sur une seule campagne. La définition de la ou des saison(s) de mort des animaux présents dans la sépulture peut donc se révéler une donnée très importante.

Quelques exemples issus des sites mongols. Les trois cas que nous avons sélectionnés pour la présentation qui suit montrent la richesse des données récoltées à partir des observations de terrain et l'importance de ces informations pour comprendre une civilisation disparue.

\section{La tombe T2o de Gol Mod}

Située dans le secteur nord de la nécropole, la tombe T2o est une sépulture monumentale (parmi les dix plus grandes du site) et flanquée à l'est de quatre tombes satellites fouillées anciennement [Fig.5]. Datée de première moitié du ${ }^{\mathrm{er}}$ siècle de notre ère, elle est surmontée d'un monument de $57 \mathrm{~m}$ de long orienté au nord et la fosse descend à 18,3 $\mathrm{m}$ de profondeur. Les dimensions de la chambre étaient de 4,2 $\mathrm{m}$ sur 2,9 m. Elle contenait des ossements de cheval et de caprinés, originellement contenus dans des récipients en alliage cuivreux. Leur situation et leur nature les définissent comme des dépôts alimentaires (selle, culotte, noix et morceau de longe).

Le dépôt le plus important se situe sur la pente nord de la fosse, à environ $8 \mathrm{~m}$ au-dessus de la chambre funéraire. C'est là qu'ont été découverts de nombreux vestiges de faune, principalement du cheval (16 individus), dans une couche de charbons et de cendres [Fig.6]. À proximité des têtes de chevaux étaient disposées 32 portions de pattes antérieures dont les phalanges étaient extrêmement abîmées, écrasées et parfois détruites. C'est le cas surtout des troisièmes phalanges. Quelques-unes d'entre elles avaient même été déformées, probablement en raison du contact de l'os avec les charbons encore chauds.

Ce type de dépôt comprenant crâne, éléments des pattes et premières côtes, étant fréquemment observé dans le monde funéraire xiongnu (Crubézy et al., 1996; Batsaïkhan 2002; Törbat, 2004, 2006; Miller et al., 2006; Tseveendorj, 1992). La position de ces vestiges dans la tombe indique clairement une volonté d'associer les animaux au défunt tout en les en isolant, un caractère que nous retrouverons aussi à Egiin Gol. Du point de vue anatomique, ces chevaux ne sont pas très grands, leur stature, d'une dizaine de centimètres supérieure à celle des chevaux mongols actuels, s'approchant de la limite supérieure de celle des chevaux de Przewalskii. On note, à leur propos, la présence d'un caractère archaïque (Prat, 1980), une p1 ou dent de loup, que l'on retrouve de façon fréquente chez les chevaux mongols actuels.

Aucune trace consécutive à la mise à mort ou encore à la découpe de la viande n'a été observée sur ces pièces. Il n'est donc pas possible de préciser si, lors du dépôt, les portions de chevaux comprenaient encore des matières organiques ou bien si les ossements avaient été entièrement ou partiellement décharnés. Toutefois, la déformation observée sur les phalanges de cheval atteste que les os étaient probablement frais au moment de leur dépôt sur les braises. Les résultats concernant les analyses cémento-chronologiques montrent que les chevaux et le bœuf ont tous été abattus au cours de la période estivale, ce qui correspond à l'actuel été mongol. Par ailleurs, la préservation de connexions labiles suggère un délai relativement court entre la mort des animaux et le placement des vestiges dans la tombe. Le nombre important des animaux déposés, ainsi que leur bon état sanitaire, infirme l'hypothèse de morts naturelles et évoquent immanquablement la notion de sacrifice. On ne peut, a priori, exclure l'éventualité d'un abattage effectué antérieurement à l'inhumation, avec conservation d'une partie du corps des animaux, pratique rendue possible à la faveur de conditions climatiques très rudes. Décès, inhumation, mise à mort du cortège animalier pouvant dans certains cas être décalés dans le temps, il peut s'avérer très important de vérifier si l'abattage des animaux associés à la tombe est intervenu au même moment que le décès du sujet. Dans cette perspective, il aurait été très utile de comparer ces résultats avec des analyses dentaires du défunt, ce qui n'a pu être fait en raison de son mauvais état de conservation. Néanmoins, la datation radiocarbone atteste qu'il ne s'est pas écoulé un long laps de temps entre les deux phénomènes.

Il est donc possible de proposer le déroulement suivant des faits : on effectue d'abord, au niveau de la chambre funéraire, un dépôt de pièces de viande de cheval et de mouton, contenu dans des récipients en alliage cuivreux, comme don 
alimentaire au défunt. Après remblai de quelques mètres au-dessus de la chambre, dans un niveau spécifiquement consacré au dépôt d'animaux sacrifiés, sur un lit de braises, on dépose un ensemble de têtes et de pattes antérieures de chevaux, évoquant des animaux en marche, ainsi qu'une tête et des pattes antérieures d'un boviné. Parmi les éléments de harnachement présents dans la chambre funéraire, à un niveau très distant et séparé de celui où se trouvaient les restes des chevaux, certaines pièces décorées d'un bestiaire semi-fantastique ont été exhumées [Fig.7]. Ainsi, on retrouve à plusieurs reprises un animal à tête de cheval, pattes d'artiodactyle et cornes de bouquetin. Ces éléments mobiliers, ainsi que les restes de chevaux sur un niveau de charbons et de cendres, sont propices à la formulation d'une interprétation symbolique, liant étroitement l'animal aux croyances xiongnu.

\section{Les sépultures $n^{\circ} 15,18$ et 19 d'Egiin Gol}

La nécropole xiongnu d'Egiin Gol, localisée à plus de $150 \mathrm{~km}$ à vol d'oiseau au nord de la nécropole de Gol Mod, a été, comme cette dernière, utilisée sur une longue période, comprise entre le $\mathrm{IV}^{\mathrm{e}}$ siècle avant notre ère et le $\mathrm{III}^{\mathrm{e}}$ siècle de notre ère. Les tombes mises au jour diffèrent largement de celles de Gol Mod, car elles semblent réservées à des individus de condition plus modeste. Toutefois, elles ont aussi livré un important matériel faunique associé qui, lui aussi, documente les rituels xiongnu et le mode de vie de ce peuple.

D’une manière générale, les tombes, délimitées à leur surface par des cercles de pierres, sont peu profondes. Les dépôts de faune les plus importants se situent sur le fond de la fosse, généralement dans une niche au nord, derrière la tête du défunt et spécialement aménagée à cet usage. Ils se trouvent ainsi associés au défunt, tout en restant à l'écart de sa dépouille, comme à Gol Mod. Cette disposition constitue l'une des caractéristiques des inhumations xiongnu. Les vestiges du remplissage de la fosse (dons ou offrandes de type alimentaire ou reliefs de repas funéraires) se distinguent de ceux placés dans les niches qui renvoient à des représentations purement symboliques, ainsi que le montrent les observations réalisées sur trois tombes, $\mathrm{n}^{\circ \mathrm{s}} 15,18$ et 19.

La tombe $\mathrm{n}^{\circ} 15$, datée entre le $\mathrm{I}^{\mathrm{er}}$ et le début du IV $v^{e}$ siècle, est orientée au nord et sa fosse, profonde de $3 \mathrm{~m}$ maximum, a livré un cercueil avec le corps d'un jeune homme, sept pointes de flèches, les restes d'un arc et, à l'extérieur du caisson proprement dit, un coffret contenant deux baguettes en os. En arrière de sa tête se trouvait une niche creusée en sape qui renfermait les dépouilles de divers animaux : des moutons, mâles et femelles, une chèvre, un bœuf et un cheval. Initialement, les peaux avaient été enroulées autour de la tête de chaque animal et étaient accompagnées des deux, voire des trois, premières paires de côtes.

La tombe $\mathrm{n}^{\circ} 18$, datée entre le $\mathrm{II}^{\mathrm{e}}$ siècle avant notre ère et le $\mathrm{I}^{\mathrm{er}}$ siècle de notre ère, dont la profondeur avoisinait 3,2 m, était également orientée au nord. Son remplissage contenait des cendres, des charbons et des sédiments rubéfiés ainsi qu'un bois de chute d'un cerf mâle adulte, dans le coin sud-ouest de la fosse, entouré de quatre petites pierres. Dans le sédiment de comblement, de toute évidence en position secondaire, des restes de pattes de caprinés et de bovinés, ainsi que de petits fragments de diaphyses d'oiseau. Toutefois, la majorité des dépôts était, là encore, située dans une niche, aménagée du côté nord de la sépulture, en arrière et séparée de la tête du sujet. Ces dépôts consistaient en des dépouilles comprenant la tête, les extrémités des pattes et la queue de 17 animaux. Comme pour la tombe $\mathrm{n}^{\circ} 15$, les dépouilles de peau, sauf deux, avaient été enroulées autour de la tête de chaque animal avant d'être déposées et les deux premières paires de côtes étaient généralement associées à ces dépôts. Des traces de découpe étaient observables. Létat sanitaire des animaux était, là encore, relativement bon et le choix des individus déposés dans la tombe ne semble donc pas s'être orienté vers des sujets affaiblis.

Il se dégage une certaine constance de l'agencement de ces dépôts. Les dépouilles des chevaux ont été placées en dernier dans la niche, des foetus animaux disposés aussitôt après : celui d'un cheval se trouvait ainsi sur un crâne de jument contre la paroi nord et c'est une disposition similaire qui a été observée également pour un fotus de boviné. Les ossements d'un troisième foetus, de capriné cette fois, étaient étroitement imbriqués dans le crâne d'une chèvre. Au-dessus du coffre contenant le squelette du défunt, une portion de coxal de foetus humain a également été retrouvée. On fait là immanquablement la relation avec les foetus animaux présents dans la même tombe.

Sur les quatre équidés retrouvés dans la niche de la tombe ${ }^{\circ} 18$, deux portaient encore des éléments de harnachements en place, en particulier le mors dans la bouche. Il en va de même pour le taureau.

Des études relatives à la saison de décès montrent que la majorité des sujets, hommes et animaux, ont trouvé la mort au cours de la période estivale. Les analyses montrent que deux bovinés sont morts à une autres saison, ce qui peut s'expliquer par des problèmes relatifs au mode d'accroissement cémentaire (ce qui renvoie à une pathologie) ou bien à des animaux morts avant l'inhumation et dont les dépouilles, conservées, ont ensuite été déposées dans la tombe. Il peut également s'agir d'animaux sacrifiés lors d'une cérémonie préalable.

Notre troisième cas, la tombe $n^{\circ} 19$, datée entre le $\mathrm{I}^{\mathrm{er}}$ siècle avant notre ère et le $\mathrm{III}^{\mathrm{e}}$ siècle de notre ère, contenait, à l'extrémité nord de la tombe, dans la niche située à la tête du défunt, un dépôt de faune vraisemblablement réalisé en trois étapes. La première étape a consisté à installer dans le coin ouest, au fond de la niche, une dépouille de boviné avec la peau enroulée autour de la ou des deux premières côtes gauches déposées sous le crâne. Dans le coin est de la niche avait été placée la dépouille d'une chèvre, crâne vers le nord, peau elle aussi roulée, contenant encore la tête et les phalanges. Dans un deuxième temps, trois dépouilles de chèvres ont été déposées, là encore, les peaux roulées 
contenant la tête et les phalanges de chaque animal. Dans un troisième temps a eu lieu le dépôt d'une pouliche au crâne disposé vers l'ouest, la peau avec la tête et les phalanges étant encore une fois roulée, et l'animal avait un mors en os dans la bouche. Le défunt est mort au cours de la bonne saison.

La projection de l'imaginaire d'un groupe humain sur l'animal se retrouve dans de nombreuses civilisations, comme le montrent les nombreux témoignages exhumés en Asie centrale, par exemple, pour l'âge du Fer, les kourganes de Pazyryk, au Kazakhstan : dans le kourgane 1, daté des $\mathrm{V}^{\mathrm{e}}-\mathrm{III}^{\mathrm{e}}$ siècles avant notre ère, on a retrouvé les restes de dix chevaux déposés à l'extérieur de la chambre funéraire (Rudenko, 1970). La tombe de Berel, non loin du site précédent, datée du début du $\mathrm{III}^{\mathrm{e}}$ siècle avant notre ère, a livré son lot de découvertes d'archéozoologie. S'agissant des sites xiongnu, à des périodes plus récentes, les animaux (en premier lieu, le cheval) sont présents de façon constante aux côtés de l'homme. Le chien, absent des tombes évoquées ici, était présent dans plusieurs cimetières et souvent enterré selon des rituels particuliers, parfois dans des cercueils en pierre (Törbat, 2004, 2006).

En règle générale, on retrouve les mêmes espèces animales et les mêmes parties des carcasses dans les différentes sépultures. Ces pratiques renvoient d'une part à des dons de type alimentaire (sous forme d'offrandes faites aux défunts ou de reliefs de repas funéraire) et, d'autre part, à une dimension purement symbolique, telle une escorte (principalement des chevaux) accompagnant le mort dans l'au-delà. Ce type de dépôt est réservé aux tombes les plus monumentales qui, à l'évidence, appartiennent à l'aristocratie, sans qu'il soit toutefois possible de les attribuer de manière spécifique aux chanyu, les chefs suprêmes. Il semble aussi qu'une hiérarchisation ait existé dans la signification accordée aux dépôts. Les chevaux, représentant les animaux les plus « nobles», étaient souvent déposés en dernier, comme à Egiin Gol, et/ou en grande quantité comme dans la tombe monumentale T2o de Gol Mod, une constatation qui ne fait que renforcer l'hypothèse d'un rôle symbolique de type psychopompe accordé à ces montures. Les sources écrites chinoises telles que les Annales historiques de Sima Qian (145-86 avant notre ère) distinguaient parfois une hiérarchie parmi les équidés eux-mêmes, précisant que certains rites nécessitaient le sacrifice d'un cheval à la robe blanche, autant d'éléments qui viennent compléter $a$ posteriori l'étude de terrain proprement dite.

Des bovinés et des caprinés sont retrouvés comme dépôts des inhumations plus modestes, faisant penser à une symbolisation concrète du cheptel du défunt. Sauf cas particuliers, des têtes ou des portions de pattes et des sacrums sont déposés, et non pas des carcasses complètes. La disposition des offrandes différait sensiblement d'un site à l'autre en fonction de la typologie de la sépulture. Ainsi, à Egiin Gol, les dépouilles étaient enroulées autour des têtes animales ou étalées et déposées en arrière, dans une niche séparée. En revanche, à Gol Mod, dans la tombe monumentale T20, les dépôts placés dans le remplissage se trouvaient à l'extérieur du sarcophage, côté nord, ce qui correspond à l'orientation cardinale des constructions de cette civilisation, observations anascaphiques confirmées par les textes. Dans les deux sites, l'une des constantes de ces offrandes demeure l'association des vestiges d'animaux avec le défunt tout en maintenant une séparation claire entre eux. Une forme de parallélisme des vestiges humains et animaux est également relevée, s'agissant en particulier des dépôts concomitants de fotus animaux et humains.

En ce qui concerne le déroulement du rituel dans le temps, l'archéozoologie apporte également des informations nouvelles. Ainsi, dans les deux sites en question, les animaux choisis pour le dépôt sont morts au cours de la bonne saison.

Par ailleurs, les dernières étapes de l'inhumation ne semblent pas avoir été différées, bien que, dans de rares cas, la mort de certains animaux puisse se situer un peu en amont du décès et de l'inhumation du sujet. L'analyse de la saison du décès n'a pu être systématiquement effectuée sur les restes humains mais, lorsque cela était possible, on constate que la mort est également survenue au cours de la bonne saison. Ce type d'étude mériterait évidemment de devenir systématique, afin de mieux apprécier la signification de ces observations et de cerner au plus près les pratiques funéraires en usage.

Enfin, des observations comparables à celles effectuées à Egiin Gol et Gol Mod ont été réalisées dans plusieurs tombes xiongnu en Mongolie et en Bouriatie, comme à Duurlig Nars, Gol Mod II, Ilmovaya Pad' Tsaram, ou Noïn Ula, pour les sites les plus fameux. Si une certaine variabilité existe dans les us, on constate une nette continuité dans les conceptions et les gestes qui en sont à l'origine. Cette similarité est d'autant plus étonnante qu'on l'observe d'ouest en est, sur l'ensemble du territoire mongol. Les vestiges d'animaux et la récurrence de leur traitement renvoient donc à des questions relatives à la composition et à l'homogénéité des groupes humains qui constituent la population xiongnu, ainsi quà leur mode de vie dont finalement rien ne concourt à montrer le caractère pleinement nomade. Les recherches font apparaître l'image d'une puissante confédération à l'intérieur de laquelle les préoccupations propres aux différents clans, aux noms bien identifiés par les chroniques antiques, vont s'inscrire de façon pérenne et avec une grande uniformité dans les pratiques funéraires.

Nous souhaitons ici remercier la commission des Recherches archéologiques à l'étranger du MAEE et le musée national des Arts asiatiquesGuimet pour leur soutien, ainsi que l'Inrap, le Centre de recherches et de restauration des Musées de France, l'Université de Bonn, l'Université Elte et le Muséum d'histoire naturelle dont le concours a été essentiel à la conduite de notre action. De même, notre gratitude s'adresse aux membres de l'équipe de la MAFM, ainsi qu'à tous ceux qui, nombreux, ont à divers titres pu contribuer à la mise en œuvre de cette étude. 


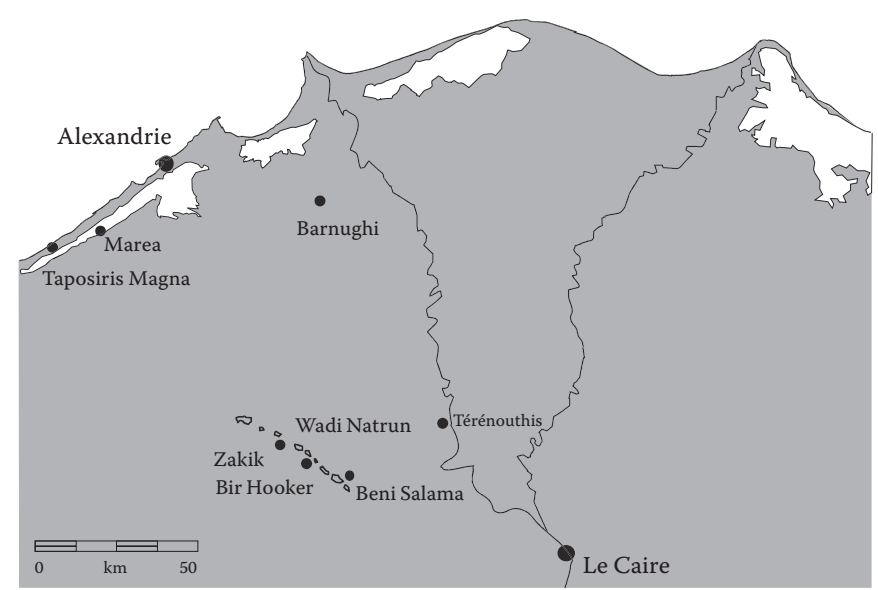

$\approx$

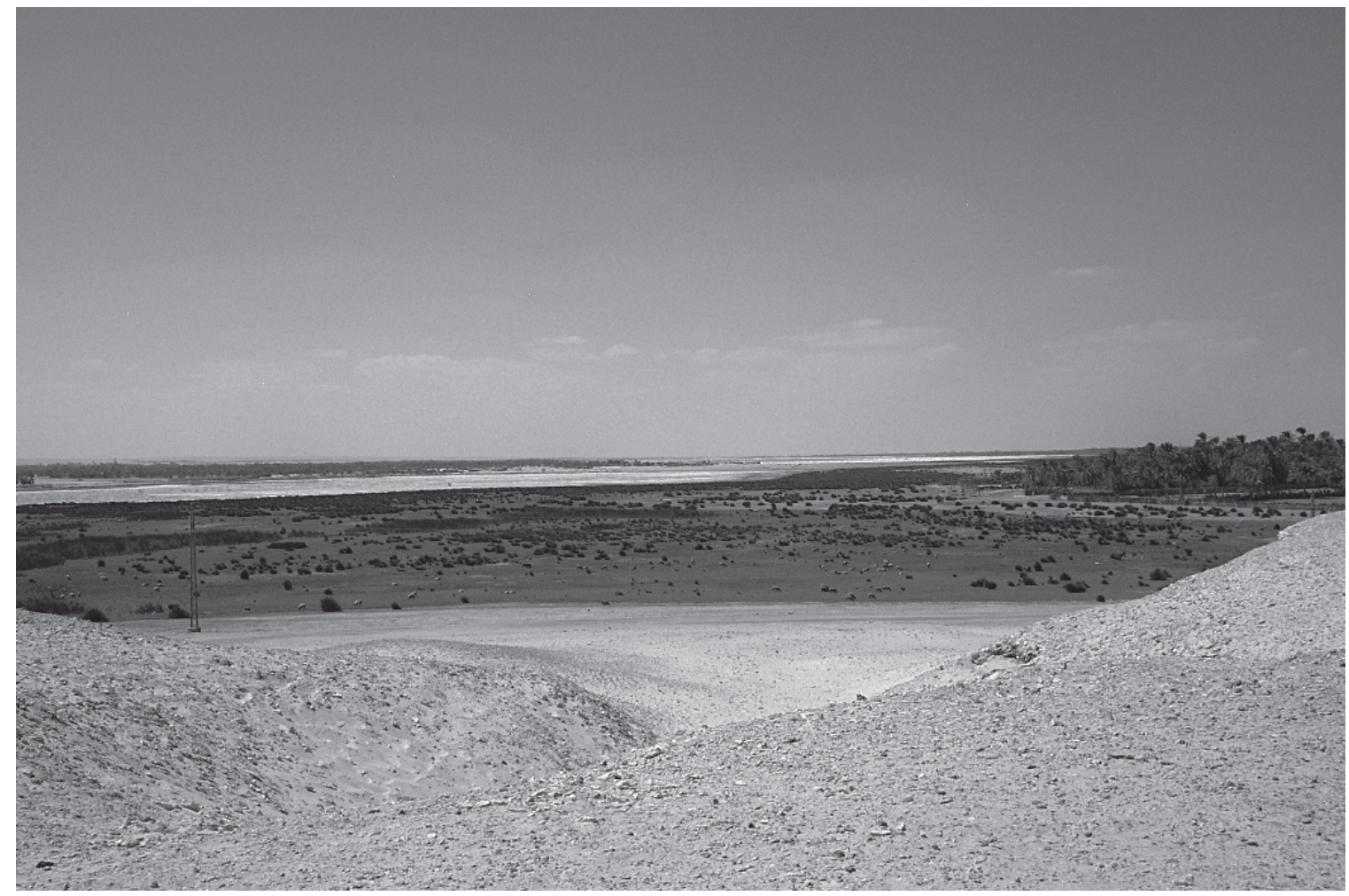

[Fig. 1] Vue du lac Al-Fasidah depuis le plateau dominant le site de Beni Salama. 
003, Mongolie, le premier empire des steppes, Paris, Actes Sud / Mafm, 244. p. ANDRE G., 2002, « Une tombe princière Xiongnu à Gol Mod, Mongolie (campagnes de fouilles 2000-2001) ", Arts Asiatiques, Paris, tome 57, p. 194-205.

Andre G., Holotova-SzineK J., 2009, « Mengu Xiongnu guizu mudi de chubu yanjiu », Kaogu Xuebao, Zhongguo Shehui Kexueyuan, Pékin, p. 69-88.

BATSAÏKHAN Z., 2002, Xiongnu : Archaeology, ethnography and history, National University of Mongolia, Oulan-Bator, édition D. Bayar, 291 p

Brosseder U., 2007, « Zur Archäologie der Xiongnu », in Attila und die Hunnen, catalogue d'exposition du musée de Speyer, Stuttgart, p. 63-71.

Crubézy E., Martin H., Giscard P.-H., Batsaikhan Z., Erdenebaatar S. MAUReille B., Verdier J.-P., 1996, « Pratiques funéraires et sacrifices d'animaux. De la représentativité au symbole. À propos d'une sépulture protohistorique de Mongolie, vallée d’Egiin Gol (région péri-Baïkal) », Paléorient, 22, p. 89-108.

Desroches J.-P. (DIR.), 2001, L'Asie des steppes d'Alexandre le Grand à Gengis khan, catalogue d'exposition, musée national des Arts asiatiques-Guimet, 23 janvier-2 avril, Paris, RMN, $191 \mathrm{p}$

Desroches J.-P., octobre-janvier 2003, «La nécropole de Gol Mod en Mongolie », in Comptes-Rendus de l'Académie des inscriptions et belles-lettres, fasc. III, De Boccard, Paris, p. 1167-1176.

Desroches J.-P., 2005, «Die Welt des Steppe - Das Reich der Xiongnu», in Dchingis Khan und Seine Erben - Das Weltreich der Mongolen, catalogue d'exposition, Bonn, 16 juin 2005- 29 janvier, p. 39-62.

Desroches J.-P., ANDre G. (ÉD.), 2007, Mongolie, les Xiongnu de l'Arkhangaï, Oulan-Bator, Admon, 97 p.

DorJsüren Ts., 2003 (réed.), Arkheologiin Sudalgaa (Mélanges d'études archéologiques), Sh.U.A., Arkheologiin Khureelen, Oulan-Bator, 376 p.

Keyser-Tracoui C., Crubézy E., Ludes B., 2003, «Nuclear and mitochondrial DNA analysis of a 2000-year-old necropolis in the Egyin Gol valley of Mongolia », American Journal of Human Genetics, 73(2), p. 247-60

Miller B., Allard F., Dimaajav E., Lee C., 2006, « A xiongnu tomb complex : excavations at Gol Mod 2 cemetery, Mongolia (20022005) », Mongolian Journal of Anthropology and Ethnology, vol. 2, $\mathrm{n}^{\circ} 2$ (271), p. 1-21.

Prat F., 1980, Les Équidés villafranchiens, CNRS, Paris, Édition du Quaternaire, $154 \mathrm{p}$

RudenKo S. I., 1970, Frozen tombs of Siberia : the Pazyryk burials of Iron-Age horsemen, Londres, J.-M. Dent and sons., 320 p.

Törbat Ts., Amartuvshin Ch., Erdenebat U., 2003, Egiin Golyn sav nutag dakhi arkheologiin dursgaluud, Oulan-Bator, Soyombo, 295 p.

TörвAт Ts., 2004, Khunnugyin jiriin iregdiin bulsh, Oulan-Bator, Soyombo, $110 \mathrm{p}$

TÖRBAT Ts., 2006, «The origin of Xiongnu archaeological culture based on funeral rites ", Mongolian Journal of Anthropology and Ethnology vol. 2, n 2 (271), p. 22-36.

TSEVEendorj D., 1992, Nomads route, International Scientific Expedition - Mongolian Archaeological, Historical and cultural Relics - Mongolia, Oulan-Bator, Ulaanbaatar Press, $430 \mathrm{p}$.

Yerööl-ERdenE CH., 2004, «Gol Modny Khünnü bulshny sudalgaany zarim ür dün», Arkheologiin suddlal, T(II) XXII, fasc. 8, Sh. U. A., Arkheologiin Khureelen, Oulan-Bator, p. 76-109.

Sous presse

Andre G., Holotova-Szinek H., Martin H., " Chevaux et Xiongnu en Mongolie : où donc trouver les cavaliers nomades?", Monumenta Serica, Sankt Augustin, p. 86-119.

Le Gall O., Demars P.-Y., Jarry M., Martin H., Saisons et proies : réflexions sur les notions de prédation(s) et de territoire. Actes du Colloque organisé dans le cadre du XVI ${ }^{\mathrm{e}}$ Congrès de l'UispP, Lisbonne, septembre 2006
Ateliers primaires de l'Égypte gréco-romaine : le site de Beni Salama dans le Wadi Natrun

Marie-Dominique Nenna

CNRS, UMR 5189 " Histoire et Sources des Mondes Antiques"

L a fouille sur les ateliers primaires du Wadi Natrun, qui a débuté en 2003 et s'est close sur le terrain en 2009, a été soutenue par le ministère des Affaires étrangères et européennes, par le laboratoire de rattachement de la directrice de la mission, Marie-Dominique Nenna (UMR 5189, Maison de l'Orient et de la Méditerranée, Lyon) et par le Centre d'études alexandrines (UsR 3134, Alexandrie) ${ }^{\mathbf{1}}$. L'Inrap y collabore par la mise à disposition d'un de ses agents, Aurore Louis, dans le cadre d'un PAS. Cette fouille vient se placer dans un programme plus large, sur lequel Marie-Dominique Nenna travaille depuis une dizaine d'années, qui vise à comprendre quel a été le rôle de l'Égypte dans l'artisanat verrier antique, aussi bien pour la production du verre brut que pour la fabrication des objets finis (vaisselle, contenants, éléments de parure et verre à vitre). Trois types de démarches se trouvent associés dans cette recherche : localisation et examen des sites de production (prospection de surface, géophysique et fouille) ; analyse archéométrique des compositions des verres bruts produits en Égypte; enfin, étude du mobilier mis au jour sur les sites de consommation.

Cadre de l'étude. Depuis une dizaine d'années, on commence à mieux comprendre le système de production et de commerce du verre dans le monde romain. Une division géographique du travail se fait entre les ateliers primaires qui produisent la matière brute à partir de deux composants, le sable et le natron (soude minérale qui permet d'abaisser la température de fusion de la silice de 1700 à $1200^{\circ} \mathrm{C}$, exploitée principalement en Égypte et notamment dans le Wadi Natrun), et les ateliers secondaires qui refondent le verre brut pour en faire des objets. Si le premier type d'atelier est attesté uniquement au Proche-Orient et en Égypte (aussi bien par les découvertes archéologiques que par les recherches archéométriques), le second est commun à l'ensemble de l'Empire romain. Il faut donc supposer un transport de verre brut (depuis les centres orientaux vers les ateliers secondaires de l'ensemble de l'Empire) qui est mis en évidence par une série de cargaisons, dont l'exemple le plus manifeste est l'épave OuestEmbiez, avec son chargement de verre brut, d'objets finis et de verre à vitre, fouillée durant la dernière décennie ${ }^{2}$.

L'intérêt pour les fours primaires des verriers antiques est récent et leur étude en est encore à ses débuts. La découverte en 1992, à Bet Eli'ezer au sud-est de Césarée, d'une série de 17 fours à bassin en a été, en grande partie, à l'origine. C'était en effet la première fois que l'on mettait au jour, et que l'on identifiait comme tels, des fours primaires.
1 Léquipe est constitué Louis (Inrap, 2004-2010), V. Merle (Inrap, 2003 (doctorante, Universite Lyon 2, 2006), V. Pichot (CNRs, Cealex, 2003 doctorante, Université Lyon 2, 2008), E. Thivet de Besançon, 2007), A. Viellet, (Service d'archéologie de Besançon, 2009) d'une architecte (J. Badr, 2007-2010), de deux céramologues, S. Marchand (Ifao, 2003-2010) et S. Marquié et d'une dessinatrice et spécialiste de vannerie (2007-2009). Les levés topographiques ont Shaalan (CNRS, Cealex) et la couverture vidéo

2 Fontaine S.-D. et Foy D., 2007, « L'épave Ouest-Embiez 1, Var : du verre brut et manufacturé en Méditerranée occidentale dans l'Antiquité », Revue archéologique de Narbonnaise, 40, p. 235-268.
GOo, "The Ancient

Glass Industry in Israel: Summary of the Finds in NenNa M.-D. (ÉD.) La Route du verre : ateliers primaires et secondaires millénaire av second au Moyen Âge, Lyon, Travaux de la Maison de l'Orient, 33, p. 49-63 FREESTONE I.C., Gorin-Rosen Y., Hughes M., «Primary Glass from Israel and the Production of Glass in Late Antiquity and the Early Islamic Period», ibidem, p. 71-72 